\title{
The Research on AUV Attitude Control based on least Squares Support Vector Machines
}

\author{
${ }^{1}$ Song Xiaoru, ${ }^{2}$ Luo Dezhu and ${ }^{3}$ Wang Huihua \\ ${ }^{1}$ School of Electronic and Information Engineering, Xi' an Technological University, \\ $X i$ 'an 710032, China; \\ ${ }^{2}$ Shaanxi electric power research institute, Xi'an 710054, China; \\ ${ }^{3}$ Xingtai Polytechnic College, Xingtai, 054035, China \\ masha0422@163.com
}

\begin{abstract}
The Attitude control model for AUV is highly nonlinear and strong coupling six degrees of freedom model. With it, and complex disturbance of the marine environment a new control strategy chaos least squares support vector machines is proposed. The control model is infinitely approximated by least squares support vector machine. The parameters are optimized Chaos theory. It is formed the Chaos-LSSVM model. The purpose is to obtain excellent attitude control of quality. The Chaos-LSSVM predictive controller for AUV attitude is designed combined with a simplified longitudinal hydrodynamic equations and the ChaosLSSVM model. Meanwhile the stability of the attitude control system is ensured by the Lyapunov stability theory. Finally, examples of simulation results show that the control model can obtain a good control effect, improve the underwater vehicle dynamic positioning attitude control effectiveness and robustness.
\end{abstract}

Keywords: AUV; Attitude Control; LSSVM; Chaos theory

\section{Introduction}

The AUV (Autonomous Underwater Vehicle) is to provide a powerful tool for deep-sea research and development resources. AUV attitude control model itself is a six-degree freedom model with highly non-linear and strong coupling. In advancing the process a wide range of model parameter perturbation can be occurred. Coupled with the difficulty of complex marine environmental interference, it is hard to the AUV attitude control. In reference [1] AUV was dynamically controlled by using a PID controller. The results are valid. But it cannot compensate for un-modeled hydrodynamic forces or unknown disturbances dynamically. And the parameters need rich experience to be determined. Reference [2] involved the Fuzzy PID controller. It was relatively simple and easy to determine the unknown parameters. However, to the parameter fluctuations the real-time robustness was not strong and the subjective factors were introduced. Fuzzy PID controller based on variable scale proposed in the reference [3]. More information can be loss by fuzzy methods. and it has greater subjective arbitrariness in the weight assignment. The sliding mode controller was introduced to control the AUV yaw control in reference [4]. The simulation results showed that the method had good robustness on the un-modeled dynamics and uncertainty environmental parameters. But there is vibration and it is offline control. Reference [5] described the NN was used in the heading control. The simulation accuracy results are satisfied. But the poor real time is a really problem, the system stability and convergence have the ascension of the larger space. 
LSSVM (Least Square Support Vector Machine) has good generalization ability, small sample of control, fast calculation speed, nonlinear system modeling. It is a powerful tool [6]. But it has a direct influence on the parameters of the control effect. The model's parameters are optimized by using COA (Chaos optimization algorithm), which is to control the attitude control model accurately. Chaos optimization algorithm is a novel optimization algorithm. It has unique in nature, such as ergodicity, randomness, regularity, non-cyclical characteristics. It has a global optimum, higher search efficiency [7]. Based on the above characteristics of the various methods, the Chaos-LSSVM attitude control system is proposed. It is to explore a new AUV attitude control system.

The rest of the paper is organized as follows. The next section is AUV simplified longitudinal motion model. The Chaos-LSSVM Model is in the section 2. The LSSVM model and the Chaos- LSSVM Predictive Model are given, respectively. The AUV attitude predictive control is designed in the section 3. It is the key part. The stability of the attitude control system is ensured by the Lyapunov stability theory in next part. In section 5 the analysis simulation results are given to demonstrate the compound Chaos-LSSVM attitude controller. In section 6, conclusions are drawn.

\section{AUV Simplified Attitude Motion Model}

According to the AUV movement law the space motion is often divided into two movement planes. They are heading motion in the lateral plane and the pitch and depth movement in the vertical plane, respectively. To facilitate research the ideal state is assumed that the roll movement is ignored in the paper. The AUV full space six degrees of freedom mode is derived in the document [2]. The AUV simplified attitude and kinematic equations are shown below.

$$
\begin{gathered}
\left\{\begin{array}{l}
\dot{\phi}=p+q \sin \phi \tan \theta+r \cos \phi \tan \theta \\
\dot{\theta}=q \cos \phi-r \sin \phi \\
\dot{\psi}=q \sin \phi / \cos \theta+r \cos \phi / \cos \theta
\end{array}\right. \\
\left\{\begin{array}{l}
\dot{X}=u \cos \psi \cos \theta+v(\cos \psi \sin \theta \sin \phi-\sin \psi \cos \phi) \\
+w(\cos \psi \sin \theta \cos \phi+\sin \psi \sin \phi)
\end{array}\right. \\
\dot{Y}=u \sin \psi \cos \theta+v(\sin \psi \sin \theta \sin \phi+\cos \psi \cos \phi) \\
+w(\sin \psi \sin \theta \cos \phi-\cos \psi \sin \phi) \\
\dot{Z}=-u \sin \theta+v \cos \theta \sin \phi+w \cos \theta \cos \phi
\end{gathered}
$$

\section{The Chaos-LSSVM Model}

\subsection{LSSVM mMdel}

Least Squares linear System is leaded in SVM. It replaces the traditional quadratic programming, aims to solve the question of the classification and function estimation. The system has the advantages of high procession-precision, stabilized performance, high speed.

LSSVM algorithm has the advantages of high procession precision and stable performance

[7]. The rule set extracted by using RS is taken as the input of LSSVM to train it. Suppose the training sample is a n-dimensional vector, $N$ samples form the sample set $D$, 
$\left\{\left(D=\left\{\left(x_{i,} y_{i}\right) \mid i=1,2, \cdots N\right\}\right), x_{i} \in R^{n} \quad y_{i} \in R\right.$, where $x_{i}$ is an input data, and $y_{i}$ the corresponding output value. LSSVM predictive regression algorithm is shown below.

$$
\hat{y}(x)=\sum_{i=1}^{N} \alpha_{i} K\left(x_{i}, x_{j}\right)+b
$$

where $K\left(x_{i}, x_{j}\right)$ is the kernel function that meets Mercer requirements. Gaussian radial basis function (RBF) is selected as the kernel function in this paper.

$$
K\left(x_{i}, x_{j}\right)=\exp \left(-\left\|x_{i}-x_{j}\right\|^{2} / 2 \delta^{2}\right)
$$

where $\delta$ is the kernel factor, i.e. the width of the kernel function.

\subsection{Chaos-LSSVM Model}

The LSSVM model's parameters are optimized by COA (Chaos optimization algorithm). The regularized parameter $C$ and the kernel factor $\delta$ are very important. The chaos theory can optimize the parameters of LSSVM model. The LSSVM parameters are acted as the combinatorial optimization problem. And then the objective function is built on it. The chaotic state brings to the optimization variables by similar carrier wave. The ergodic scope of chaotic motion extends to the upper and lower limits of the optimizing variable. A more detailed explanation follows below. Define the optimization problem as

$$
\begin{array}{r}
\min J=\min f\left(z_{i}\right)=\frac{1}{L} \sum_{i=1}^{N}\left(y_{i}-\hat{y}_{i}\right)^{2} \\
\text { s.t. } C \in\left[C_{\min }, C_{\max }\right], \sigma \in\left[\sigma_{\min }, \sigma_{\max }\right]
\end{array}
$$

where $y_{i}$ is the $i$-th known sample output value of the samples $\left\{x_{i}, y_{i}\right\}_{i=1}^{N}, \hat{y}_{i}$ is the output prediction of the former, which can be calculated from equation (11), $f\left(z_{i}\right)$ is the regression of LSSVM, $z_{i}$ is the optimized variable.

Logistic mapping is chosen to produce the chaos variables.

$$
x_{n+1}=\mu x_{n}\left(1-x_{n}\right)
$$

where the initial value $x_{0} \in(0,1)$, and $x_{0}$ is not equal to $0.25,0.5$ or 0.75 .

Logistic mapping is a full mapping in interval $(0,1)$ when $\mu=4$, a system goes into chaos state. $x_{n}$ is the ergodic in the interval $(0,1)$.

It can be seen from (11) and (12) that the objective function $J$ of the optimization problem (13) is the function of $C$ and $\delta$. $J$ will decrease and converge to its minimum $J^{*}$ if searching ergodicly in $C$ and $\delta$ definition domains by using the chaos variable. The corresponding minimum $C^{*}$ and $\delta^{*}$ will be the optimal parameters of the LSSVM.

\section{The Design on the Chaos-LSSVM Predictive Controller}

Assuming AUV constraints nonlinear systems is expressed as the following.

$$
\left\{\begin{array}{l}
x(k+1)=f(x(k), \delta(k)) \\
\text { s.t. } x(k) \in X, \delta(k) \in U
\end{array}\right.
$$


where $x(k)$ and $\delta(k)$ is the position attitude state quantity vectors and the control vector, respectively. In the case of AUV longitudinal movement, $x(k)$ is the AUV depth and pitch angle etc state quantity vectors. $\delta(k)$ is the horizontal rudder control angle. It is the depth controlled variable. Where $X$ and $U$ is defined $0 \subset X \subseteq R^{n}, 0 \subset U \subseteq R^{m}$, respectively. And both are compact and convex set. $f: R^{n} \times R^{m} \rightarrow R^{n}$, it has a continuous second-order partial derivatives. And $f(0,0)=0$. The model $x^{m}(k+1 \mid k)$ is built by the Chaos-LSSVM method. Where the superscript $\mathrm{m}$ represents a comprehensive LSSVM model. It is to overcome modeling errors and noise By introducing feedback. Assuming the state is measured, and then the output of predictive control system corrected feedback is as following.

$$
x(k+1 \mid k)=x^{m}(k+1 \mid k)+x(k)-x^{m}(k \mid k-1)
$$

The control objective is usually driven to the origin status or some equilibrium state $x_{r} \in R^{n}$. It is the former in the paper. And it meets the state and the input constraint of the equation. Suppose the any positive definite symmetric matrix is $\mathrm{S}$. the weighted norm is defined as $\|x\|_{S}^{2}=x^{T} S x . x$ is the corresponding dimension real vector. The predictive control optimization problem can be expressed as follows.

$$
\left\{\begin{array}{l}
\min J(k)=\sum_{i=0}^{H_{P}-1}\|x(k+i \mid k)\|_{Q}^{2}+\sum_{j=0}^{H_{U}-1}\|u(k+j)\|_{R}^{2} \\
\text { s.t. } \quad x(k+i \mid k) \in \mathrm{X}, \quad u(k+j) \in \mathrm{U}, i=0,1, \cdots, H_{P}-1, j=0,1, \cdots, H_{U}-1
\end{array}\right.
$$

where $x(k \mid k) \in x(k), Q$ and $P$ are symmetric positive definite matrix. $H_{P}$ and $H_{U}$ is the predictive and control time domain. And the $H_{P} \geq H_{U}$. In order to facilitate discussion it is assumes that $H_{P}=H_{U}=N$. The equation (10) is expressed is:

$$
\left\{\begin{array}{l}
\min J(k)=\sum_{i=0}^{N-1}\left(\|x(k+i \mid k)\|_{Q}^{2}+\|u(k+i)\|_{R}^{2}\right) \\
\text { s.t. } x(k+i \mid k) \in \mathrm{X}, \quad u(k+i) \in \mathrm{U}, \quad i=0,1, \cdots, N
\end{array}\right.
$$

The Chaos-LSSVM constrained nonlinear predictive control is to solve optimization problems formula (11) at the $\mathrm{k}$ moment. The optimal control law $\left\{u^{*}(k), \cdots, u^{*}(k+N-1)\right\}$ can be obtained. And its first component $u^{*}(k)$ is applied to the system. At the next moment, suppose the $k=k+1$. Then repeat the process until the end of the control.

\section{Stability Analysis of the Chaos-LSSVM Controller}

The stability is the primary control performance requirements. Given the controlled object AUV is typical nonlinear. The Chaos-LSSVM predictive control system stability is proved by Lyapunov stability theory. It is attach a terminal constraint after conventional performance indicators. And certain conditions are met. It is can be attained the system stability. Inspired by literature [9] the formula (11) can be rewrite: 


$$
\left\{\begin{array}{l}
\min J(k)=\sum_{i=0}^{N-1}\left(\|x(k+i \mid k)\|_{Q}^{2}+\|u(k+i)\|_{R}^{2}\right)+\|x(k+N \mid k)\|_{P}^{2} \\
\text { s.t. } x(k+i \mid k) \in \mathrm{X}, \quad u(k+i) \in \mathrm{U}, \quad i=0,1, \cdots, N-1 ;
\end{array}\right.
$$

where, the $x(k+N \mid k)$ is the terminal state, $x(k+N \mid k) \in \mathrm{X}_{f} \cdot Q$ and $R$ are the same meaning formula (11). $P$ is the symmetric positive definite matrix. It is also called terminal punishment array. $X_{f}$ is terminal domain constraints, $\{0\} \subset X_{f} \subset X$. Suppose $X_{f}$ state can be driven in $\mathrm{N}$ steps or less within the control of step. And the state and control constraints can be met. The local linear feedback control internally.

$$
u(k)=g(x(k))=X_{f} x(k)
$$

It can be driven to the origin of the final state. Where $g(\cdot)$ show the feedback control law. $X_{f}$ is the state feedback matrix.

Lemma 1 (Lyapunov Stability theorem) If there exists a state $x(k)$ and the scalar function $V(x(k), u(k))$ of the control $u(k)$ in the nonlinear system (8). It meet the following formula to the arbitrary $x(k) \in R^{n}, u(k) \in R^{m}$.

(1) $V(x(k), u(k))$ is positive definite matrix.

(2) Denoted $\Delta V(x(k), u(k))=V(x(k+1), u(k+1))-V(x(k), u(k)), \Delta V(x(k), u(k))$ is negative definite matrix.

(3) When $\|x(k)\| \rightarrow \infty,\|u(k)\| \rightarrow \infty$, then $V(x(k), u(k)) \rightarrow \infty$;

So the origin equilibrium state $x(k)=0, u(k)=0$ are the wide range of asymptotic stability.

Lemma 2 there exist $\alpha \in(0, \infty)$ it make the origin neighborhood $\Omega_{\alpha}=\left\{x \in R^{n}\|x\|_{P}^{2} \leq \alpha\right\}$ has the properties:

(1) $\Omega_{\alpha}$ is control invariant set of the system (8) under the control law (13) work.

(2) If the control law (13) then $\lim _{k \rightarrow \infty} x(k)=0, \lim _{k \rightarrow \infty} u(k)=0$ for the any $x(0) \in \Omega_{\alpha}$.

A wide range of asymptotic stability in the lemma 1is the global asymptotic stability. It is namely $\forall x(0) \neq 0 \in R^{n}, \quad u(0) \in R^{m}, \quad x(k)=0, \quad u(k)=0$ is the asymptotic stability. It is always wanted the system asymptotically stable with a wide range of properties on the project. However, if a system has such a property, completely determined by the structure and parameters of the system. The lemma 2 descript there exists a origin neighborhood. It is not just control invariant set, and it is asymptotically stable domain of attraction.

The so-called asymptotic stability domain of attraction refers to a set. When the initial state is located within the collection, the closed-loop system is asymptotically stable. These concepts laid a solid foundation for the following stability theorem.

Theorem 1: It can drive $x(k) \in X$ to $x(k) \in X_{f}$ within the $\mathrm{N}$ or less control steps. The local controller $u(k)=g(x(k))$ in the equation (13) in the $X_{f}$. If they can meet the following conditions.

(1) $\{0\} \subset X_{f} \subseteq X, X_{f}$ is the closed set.

(2) $g(x(k)) \in U, \forall x(k) \in X_{f}$; 
(3) $f(x(k), g(x(k))) \in X_{f}, \quad \forall x(k) \in X_{f}$;

(4) $\|f(x(k), g(x(k)))\|_{P}^{2}+\left(\|x(k)\|_{Q}^{2}+\|g(x(k))\|_{R}^{2}\right)-\|x(k)\|_{P}^{2} \leq 0, \quad \forall x(k) \in X_{f}$;

Then the origin equilibrium $x(k)=0, u(k)=0$ are the wide range of asymptotic stability

Prove: set the optimal value $J^{*}(k)$ is the Lyapunov candidate function because of the objective function $x(k), u(k)$ of the optimization problem (12). Just to prove it is to meet the three conditions in Lemma 1 . Because $P, Q$ and $R$ are symmetric positive definite matrix it is easily obtained.

(1) $J^{*}(k)$ is the positive definite.

(3) when $\|x(k)\| \rightarrow \infty,\|u(k)\| \rightarrow \infty$, then $J^{*}(k) \rightarrow \infty$.

Let's start proved equally satisfy the second conditions of Lemma 1.

The optimal control law of $J(k)$ is denoted $\left\{u^{*}(k), \cdots, u^{*}(k+N-1)\right\}$. The corresponding optimal state trajectory is denoted $\left\{x^{*}(k), \cdots, x^{*}(k+N-1)\right\}$. The optimal first component of control solution $u^{*}(k)$ is applied to the system at the $k$ moment when ignore modeling error and noise. The $k+1$ moment system state is $x^{*}(k+1)=f\left(x^{*}(k), u^{*}(k)\right)$. Similarly the $k+N$ moment system state is $x^{*}(k+N)=f\left(x^{*}(k+N-1), u^{*}(k+N-1)\right)$. The local linear control $u(k+N)=g(x(k+N))$ is used. And then the state is located in the $X_{f}$ by the known conditions. $X_{f}$ must be invariant set in the control system when control law is $g(\cdot)$.according to the known condition (1)、 (2)、(3) and the definition 2 . So $\left\{u^{*}(k+1), \cdots, u^{*}(k+N-1), g\left(x^{*}(k+N)\right)\right\}$ is a feasible solution of $J(k+1)$. The corresponding state trajectory is $\left\{x^{*}(k+1), \cdots, x^{*}(k+N), f\left(x^{*}(k+N), g\left(x^{*}(k+N)\right)\right\}\right.$.

So $J^{*}(k)$ and $J(k+1)$ can be rewrite respectively

$$
\begin{gathered}
J^{*}(k)=\left(\left\|x^{*}(k)\right\|_{Q}^{2}+\left\|u^{*}(k)\right\|_{R}^{2}\right)+\left(\left\|x^{*}(k+1 \mid k)\right\|_{Q}^{2}+\left\|u^{*}(k+1)\right\|_{R}^{2}\right)+\cdots \\
+\left(\left\|x^{*}(k+N-1 \mid k)\right\|_{Q}^{2}+\left\|u^{*}(k+N-1)\right\|_{R}^{2}\right)+\left\|x^{*}(k+N \mid k)\right\|_{P}^{2} \\
J(k+1)=\left(\left\|x^{*}(k+1 \mid k)\right\|_{Q}^{2}+\left\|u^{*}(k+1)\right\|_{R}^{2}\right)+\cdots+\left(\left\|x^{*}(k+N-1 \mid k)\right\|_{Q}^{2}+\left\|u^{*}(k+N-1)\right\|_{R}^{2}\right) \\
+\left(\left\|x^{*}(k+N \mid k)\right\|_{Q}^{2}+\left\|g\left(x^{*}(k+N \mid k) \|_{R}^{2}\right)+\right\| f\left(x^{*}(k+N \mid k), g\left(x^{*}(k+N \mid k)\right)\right) \|_{P}^{2}\right.
\end{gathered}
$$

Comparison of formula (14) and (15), it can be seen

$$
\begin{aligned}
J(k+1) & =J^{*}(k)-\left(\left\|x^{*}(k)\right\|_{Q}^{2}+\left\|u^{*}(k)\right\|_{R}^{2}\right)-\left\|x^{*}(k+N \mid k)\right\|_{P}^{2} \\
& +\left(\left\|x^{*}(k+N \mid k)\right\|_{Q}^{2}+\| g\left(x^{*}(k+N \mid k) \|_{R}^{2}\right)\right. \\
& +\left\|f\left(x^{*}(k+N \mid k), g\left(x^{*}(k+N \mid k)\right)\right)\right\|_{P}^{2}
\end{aligned}
$$

After three terms in equation (16) is less than or equal to 0 by the condition (4). Namely, 


$$
\begin{aligned}
& \left\|f\left(x^{*}(k+N \mid k), g\left(x^{*}(k+N \mid k)\right)\right)\right\|_{P}^{2}+\left(\left\|x^{*}(k+N \mid k)\right\|_{Q}^{2}+\| g\left(x^{*}(k+N \mid k) \|_{R}^{2}\right)\right. \\
& -\left\|x^{*}(k+N \mid k)\right\|_{P}^{2} \leq 0
\end{aligned}
$$

It can be easily obtain from equation (16) and (17).

$$
J(k+1) \leq J^{*}(k)-\left(\left\|x^{*}(k)\right\|_{Q}^{2}+\left\|u^{*}(k)\right\|_{R}^{2}\right)
$$

$J(k+1)$ must be the an upper bound of $J^{*}(k+1)$ according to the principle of optimality. So

$$
J^{*}(k+1) \leq J^{*}(k)-\left(\left\|x^{*}(k)\right\|_{Q}^{2}+\left\|u^{*}(k)\right\|_{R}^{2}\right)
$$

Given the matrix $Q$ and $R$ are symmetric positive definite matrix, namely it is

$$
\left(\left\|x^{*}(k)\right\|_{Q}^{2}+\left\|u^{*}(k)\right\|_{R}^{2}\right)>0
$$

So

$$
J^{*}(k+1)-J^{*}(k)<0
$$

Note $\Delta J^{*}(k)=J^{*}(k+1)-J^{*}(k)$, it can be known $\Delta J^{*}(k)$ is negative definite. It is the condition 2 in the lemma 1 is also true.

Summary the three conditions in lemma 1 are all met. So it can be seen from Lemma 1 the origin equilibrium $x(k)=0, u(k)=0$ must be a wide range of asymptotically stable. Theorem is proved.

\section{Stability Analysis of the Chaos-LSSVM Controller}

Take a certain AUV attitude predicts control as examples, the Chaos-LSSVM attitude controller is designed. The formula (1) and (2) are the AUV attitude control model. The AUV attitude angle $\eta=\left[\begin{array}{lll}\phi & \theta & \psi\end{array}\right]^{T}$ is the output value in the paper. The input one is the control rudder $u=\left[\begin{array}{ll}\delta_{e} & \delta_{r}\end{array}\right]^{T}$. At the same time the former attitude angle information is the desired attitude information. The control input value can be attained by the Chaos-LSSVM control algorithm. Namely the AUV control is achieved by controlling rudder and propeller together to complete.

The AUV run at a given constant sailing speed $u=2 \mathrm{~m} / \mathrm{s}$ in the exam. In the AUV depth control simulation the initial depth is underwater 10 meters. The reference target depth is underwater 20 meters. The desired attitude angle are $\left[\begin{array}{lll}\phi & \theta & \psi\end{array}\right]^{T}=\left[\begin{array}{lll}0 & 0 & 0\end{array}\right]$. The initial heading angle is zero. The range of horizontal and vertical rudder are $\left[-15^{\circ} \sim+15^{0}\right]$. The RBF function is the Chaos-LSSVM Kernel one by the suitable sample data. The range of parameters are $C \in[1,1000], \sigma \in[0.1,10]$. The optimization number is $A_{1}=2000$. The design parameters are $C=1000, \sigma=0.7$, and the LSSVM corresponding model parameters $a_{i}$ and $b$. The control model is the Chaos-LSSVM. The control performance is tested. The sampling period $T=0.1 s$ in the simulation. At first from the initial depth 10 meters underwater to the desired underwater 20 meters the AUV simulation results under the ChaosLSSVM control model are the Figure 1 to 3. 


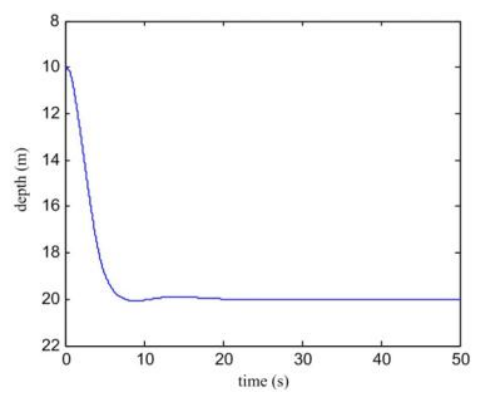

Figure 1. The Depth Change Curve

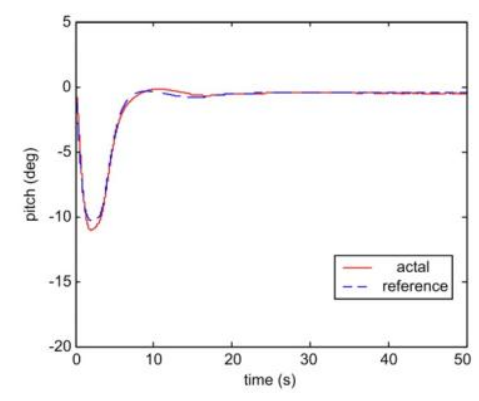

Figure 2. The Pitch Angle Change Curve

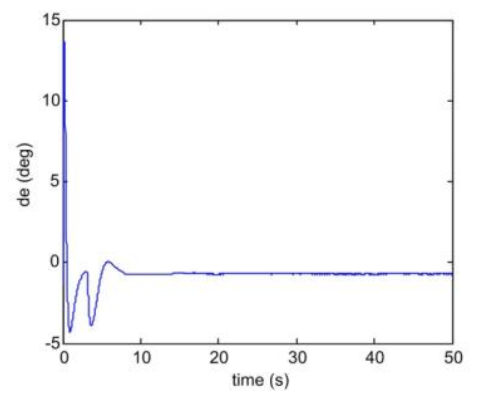

Figure 3. The Horizontal Rudder Angle Curve

The AUV depth change curve from the initial depth 10 meters underwater to the desired underwater 20 meters in the Figure 1. It is seen from the final depth stable error substantially is zero from the figure. It is illustrated the Chaos-LSSVM algorithm can reduce the control system uncertainty. It meets stable error requirements. The corresponding pitch and the horizontal rudder angle curve from 10 meters underwater to 20 in the figure 2 and 3, respectively. Thus it can be seen Chaos-LSSVM can online compensate for nonlinear uncertainty parameter. It can reduce the input control system requirements uncertainty. At the same time the AUV attitude control is studied when it steady sailing 20 meters underwater. The AUV attitude angles and rudder angles curves are showed in the figure 4 to 6 when from the 350 s to 400 s when it steady hailing.

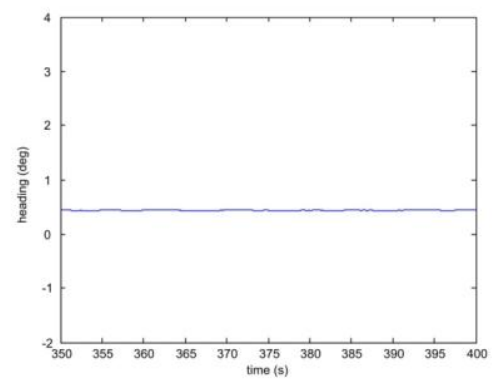

(a) The AUV stable direct sailing heading curve

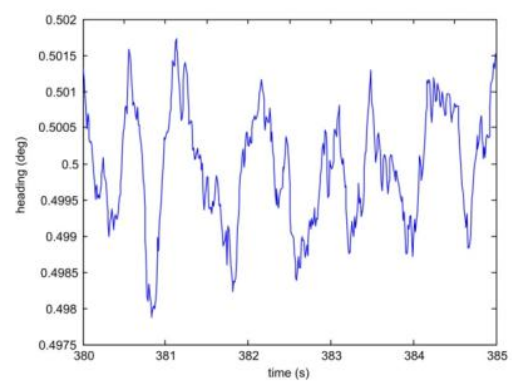

(b) the local amplification heading noise curve

Figure 4. AUV Stable Direct Route Heading Curve 


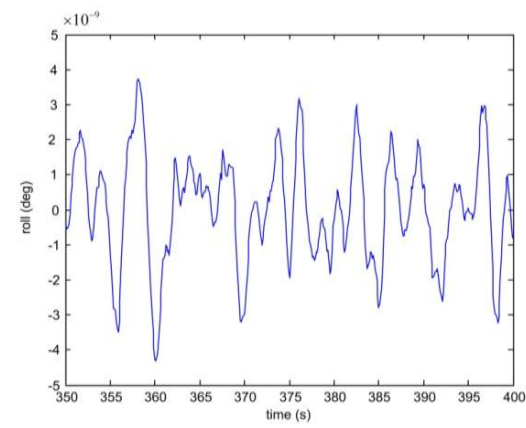

Figure 5. AUV Stable Direct Sailing Roll Curve

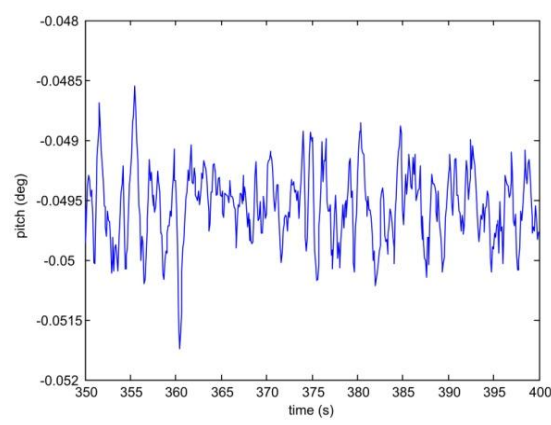

Figure 6. AUV Stable Direct Sailing Pitch Curve

The AUV stable direct response curves under the Chaos-LSSVM attitude controller from the Figure 4 to 6 . The AUV yaw is relatively small in the figure 4 . It is about plus or minus 0.5 degree error. It is approximate no difference in the direct flight. The roll and pitch curve of the AUV stable direct sailing is showed in the Figure 5 and 6. It can be seen the two errors smaller than former. It is about the percentile and micrometer error. So the Chaos-LSSVM attitude control accuracy is ideal. It achieved the desired goal.

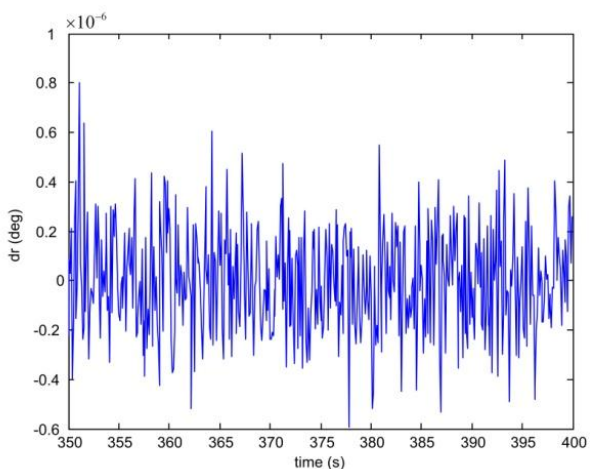

Figure 7. The Stable Direct Route Vertical Rudder Curve

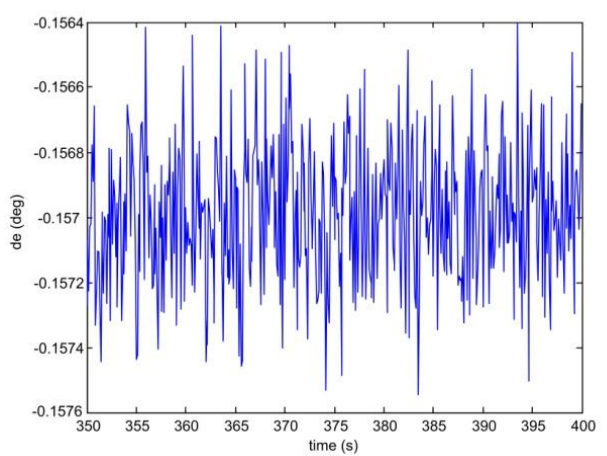

Figure 8. The Stable Direct Route Horizontal Rudder Curve

The vertical and horizontal rudder angle curves of the AUV stable direct sailing is showed in the Figure 7 and 8, respectively. The control rudder is relatively stable when the AUV constant speed directs sailing. The control effect is good.

Based on the above simulation results it can be seen the fast and accurate control is satisfactory to the Chaos-LSSVM attitude controller. So the controller has the controller has the ideal dynamic and static performance during the AUV constant speed directs sailing. It gained access to a high quality control.

\section{Conclusions}

A new Chaos-LSSVM control strategy is brought out according to the AUV nonlinear model, coupling and uncertainty etc characteristics. LSSVM can be to better solve practical problems of small samples, nonlinearity, high dimension and local minima. It is a powerful tool for nonlinear system modeling. Therefore LSSVM can infinite approximation model. Chaos theory has ergodicity, randomness, regularity, non-cyclical characteristics. It has global 
optimum and higher search efficiency. Therefore chaos theory is to optimize its parameters. It made the good quality of the attitude control. The simulation results show that the control method can obtain a high quality control. For model uncertainty is robust and certain antijamming capability. Finally the simulation results examples show that the control model has obtained a good control effect. It is effectively improve the AUV motion control effectiveness and robustness.

\section{Acknowledgements}

The project is financially supported the Education Department of Shaanxi Provincial Government Research Project (14JK1336) and the Xi'An Science and Technology Bureau Project. The authors would like to thank the underwater vehicle Institute of Marine Engineering, Northwestern Polytechnical University. The parts of experiment have been done by their help and support. And the authors would like to express my gratitude to my friends who have helped me!.

\section{References}

[1] O. Alec and McD. Make, "Failure detection in an autonomous underwater vehicle [A]", Proceedings of the IEEE Symposium on Autonomous Underwater Vehicle Technology [C], (1998), pp. 377-382.

[2] V. Kanakakis and K. P. Valavanis, "Fuzzy Logic Navigation of Underwater Vehicles [J]", Journal of Intelligent and Robotic Systems, (2004), pp. 45-88.

[3] T. Motoyuki and U. Tamaki, "Development of a system to diagnose autonomous [J]", International Journal of Systems Science, vol. 30, no. 9, (2003), pp. 981-988.

[4] A. J. Healey and D. Lienard, "Multivariable sliding mode control for autonomous diving and steering of unmanned underwater vehicles [J]", IEEE Journal of Oceanic Engineering, vol. 18, no. 3, (2008), pp. 327-339.

[5] J. Juh, "A neural net controller for underwater robotic vehicles [J]", IEEE Journal of Oceanic Engineering, vol. 15, no. 3, (2006), pp. 161-166.

[6] H. Shayeghi and A. Ghasemi, "Day-ahead electricity prices forecasting by a modified CGSA technique and hybrid WT in LSSVM based scheme [J]", Energy Conversion and Management, vol. 74, (2013), pp. $482-491$.

[7] W. C. Hong, "Application of chaotic ant swarm optimization in electric load forecasting [J]", Energy Policy, vol. 38, no. 10, (2010), pp. 5830-5839.

[8] C.-W. Chen, J.-S. Kouh and J.-F. Tsai, "Modeling and Simulation of an AUV Simulator with Guidance System [J]", IEEE Journal of Oceanic Engineering, vol. 38, no. 2, vol. 4, (2013), pp. 211-225.

[9] C. E. Garcia and M. Morari, "Internal model control a flexible algorithm for the computer control of industrial processes", 1981 International Conference on Computerized Control and Operation of Chemical Plants. IEEE Press, (1981), pp. 32-38.

[10] D. Q. Mayne and J. B. Rawings, "Constrained model predictive control: Stability and optimality [J]", Automatic, vol. 36, no. 6, (2000), pp. 789-814.

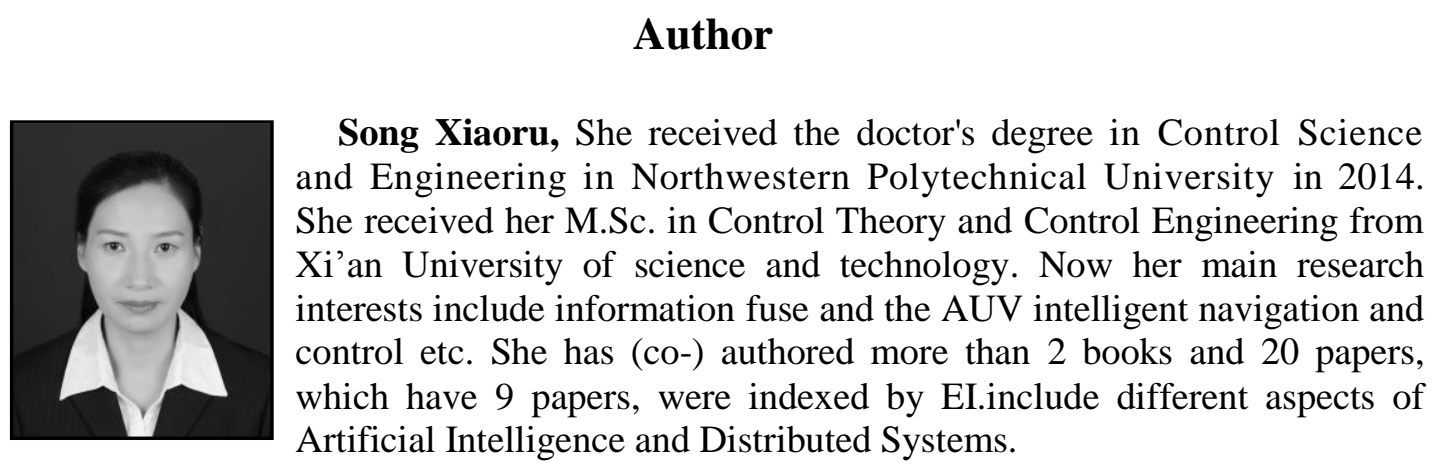

